\title{
Maximal fillings of moon polyominoes, simplicial complexes, and Schubert polynomials
}

\author{
Luis Serrano \\ LaCIM \\ Université du Québec à Montréal \\ Montréal, QC, Canada \\ serrano@lacim.ca
}

\author{
Christian Stump \\ LaCIM \\ Université du Québec à Montréal \\ Montréal, QC, Canada \\ christian.stump@lacim.ca
}

Submitted: Jul 8, 2011; Accepted: Jan 2, 2012; Published: Jan 16, 2012

Mathematics Subject Classification: Primary 05E45; Secondary 05A05, 05 E05

\begin{abstract}
We exhibit a canonical connection between maximal $(0,1)$-fillings of a moon polyomino avoiding north-east chains of a given length and reduced pipe dreams of a certain permutation. Following this approach we show that the simplicial complex of such maximal fillings is a vertex-decomposable, and thus shellable, sphere. In particular, this implies a positivity result for Schubert polynomials. Moreover, for Ferrers shapes we construct a bijection to maximal fillings avoiding south-east chains of the same length which specializes to a bijection between $k$-triangulations of the $n$-gon and $k$-fans of Dyck paths of length $2(n-2 k)$. Using this, we translate a conjectured cyclic sieving phenomenon for $k$-triangulations with rotation to the language of $k$-flagged tableaux with promotion.
\end{abstract}

\section{Introduction}

Fix positive integers $n$ and $k$ such that $2 k<n$. A $k$-triangulation of a convex $n$-gon is a maximal collection of diagonals in the $n$-gon such that no $k+1$ diagonals mutually cross. A $k$-fan of Dyck paths of length $2 \ell$ is a collection of $k$ Dyck paths from $(0,0)$ to $(\ell, \ell)$ which do not cross (although they may share edges).

The following theorem is the first main result in this article. It answers a question in R. Stanley's Catalan Addendum [Sta11], and extends results by S. Elizalde [Eli07] and C. Nicolás [Nic09].

Theorem 1.1. There is an explicit bijection between $k$-triangulations of a convex $n$-gon and $k$-fans of Dyck paths of length $2(n-2 k)$. 
A north-east chain of length $\ell$ in a Ferrers shape $\lambda$ is a sequence of $\ell$ boxes in $\lambda$ such that every box in the sequence is strictly north and strictly east of the preceding one, and for which the smallest rectangle containing all boxes in the sequence is also contained in $\lambda$. A $k$-north-east filling of $\lambda$ is a $(0,1)$-filling which does not contain any north-east chain of 1 's of length $k+1$, and in which the number of 1's is maximal. As usual, we identify a $(0,1)$-filling with its set of boxes filled with 1's and draw them by marking its set of boxes by + 's. See Figure 1(a) for an example. The set of all $k$-north-east fillings of $\lambda$ is denoted by $\mathcal{F}_{N E}(\lambda, k)$. South-east chains, $k$-south-east fillings and $\mathcal{F}_{S E}(\lambda, k)$ are defined similarly.

It is well-known that $k$-triangulations of the $n$-gon can be seen as $k$-north-east fillings of the staircase shape $(n-1, \ldots, 2,1)$, and furthermore, $k$-fans of Dyck paths of length $2(n-2 k)$ can be seen as $k$-south-east fillings of the same staircase (see e.g. [Kra06a, Rub06]). Thus, the second main theorem is a clear extension of the first. It answers a questions raised by C. Krattenthaler in [Kra06a].

Theorem 1.2. Let $\lambda$ be a Ferrers shape and let $k$ be a positive integer. There is an explicit bijection between $k$-north-east and $k$-south-east fillings of $\lambda$.

The constructed bijection goes through two intermediate objects, namely through pipe dreams and flagged tableaux, both arising in the theory of Schubert polynomials. The third main theorem is a central step in the proof of Theorem 1.2 and it concerns the connection between north-east chains and reduced pipe dreams.

Theorem 1.3. Let $\lambda$ be a Ferrers shape and let $k$ be a positive integer. There exists a canonical bijection between $k$-north-east fillings of $\lambda$ and reduced pipe dreams of a permutation depending on $\lambda$ and $k$.

This bijection will be described in Section 2. A variation of the argument gives the following generalization to moon polyominoes as defined in Section 2.2.

Theorem 1.4. Let $M$ be a moon polyomino and let $k$ be a positive integer. Then there exists a canonical bijection between $k$-north-east fillings of $M$ and reduced pipe dreams (of a given permutation) living inside $M$.

We will use the construction to obtain new properties and simple proofs for known properties of $k$-north-east fillings and of $k$-triangulations. In particular, we obtain the following corollaries.

Corollary 1.5. The simplicial complex with facets being $k$-north-east fillings of a moon polyomino $M$ is the join of a vertex-decomposable, triangulated sphere with a full simplex. In particular, it is shellable and Cohen-Macaulay.

Corollary 1.6. Let $S$ be a stack polyomino and $\lambda$ the Ferrers shape obtained from $S$ be properly rearranging its columns. Let $\sigma$ and $\tau$ be the associated permutations, depending on the shape of $S$, and $\lambda$ (see Section 2.2). Then the difference

$$
\mathfrak{S}_{\sigma}\left(x_{1}, x_{2}, \ldots\right)-\mathfrak{S}_{\tau}\left(x_{1}, x_{2}, \ldots\right)
$$

of Schubert polynomials is monomial positive. 
The bijection for $k$-triangulations has the additional property that the cyclic action given by rotation of the $n$-gon corresponds to a promotion-like operation on flagged tableaux and thus transforms a conjectured cyclic sieving phenomenon (CSP) into the context of $k$-flagged tableaux.

Conjecture 1.7. Let $\mathcal{F} \mathcal{T}(\lambda, k)$ be the set of $k$-flagged tableaux and let $\rho$ be the promotionlike cyclic action on $\mathcal{F} \mathcal{T}(\lambda, k)$. The triple

$$
(\mathcal{F} \mathcal{T}(\lambda, k),\langle\rho\rangle, F(q))
$$

exhibits the CSP, where

$$
F(q):=\prod_{1 \leq i \leq j<n-2 k} \frac{[i+j+2 k]_{q}}{[i+j]_{q}}
$$

is a natural q-analogue of the cardinality of $\mathcal{F}_{N E}(\lambda, k)$.

\section{From north-east fillings to pipe dreams}

In this section we exhibit a connection between $k$-north-east fillings of Ferrers shapes as well as of stack and moon polyominoes on the one hand and reduced pipe dreams on the other. This generalizes a construction by the second author for $k$-triangulations [Stu10], and by V. Pilaud and M. Pocchiola [Pil10, PP10], where they refer to pipe dreams as pseudoline arrangements.

Reduced pipe dreams (or $r c$-graphs) were introduced by S. Fomin and A. Kirillov in [FK96] (see also work of N. Bergeron and S. Billey [BB93]). They play a central role in the combinatorics of Schubert polynomials of A. Lascoux and M.-P. Schützenberger. A pipe dream of size $n$ is a filling of the staircase shape $(n-1, \ldots, 2,1)$ where each box contains two crossing pipes + or two turning pipes r. See Figure 1(b) for an example. A pipe dream is identified with its set of boxes containing two crossing pipes + . The permutation $\pi(D)$ of a pipe dream $D$ is obtained by following the pipes starting from the top and going all the way to the left, labelling the numbers on the left column with respect to the opposite end of the corresponding pipe, and then reading $\pi(D)$ on the left from top to bottom in one line notation. For example, the permutation of the pipe dream in Figure 1(b) is $[1,2,7,6,5,8,3,4,9,10]$. A pipe dream is reduced if two pipes cross at most once. We say that a pipe dream lives inside a set $M$ of boxes in the staircase shape if all its crossings are contained in $M$. For a given permutation $\pi$ and a set $M$ of boxes, denote the set of reduced pipe dreams for $\pi$ by $\mathcal{R P}(\pi)$ and the set of reduced pipe dreams for $\pi$ which live inside $M$ by $\mathcal{R} \mathcal{P}(\pi, M)$.

\subsection{A bijection between north-east fillings and reduced pipe dreams}

Starting with a $k$-north-east filling of $\lambda$, one obtains a pipe dream by replacing every 1 by two turning pipes and every 0 by two crossing pipes. Afterwards, $\lambda$ is embedded into the 


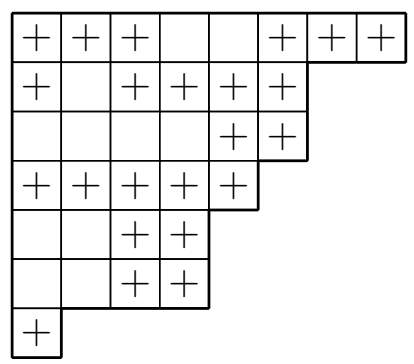

(a)

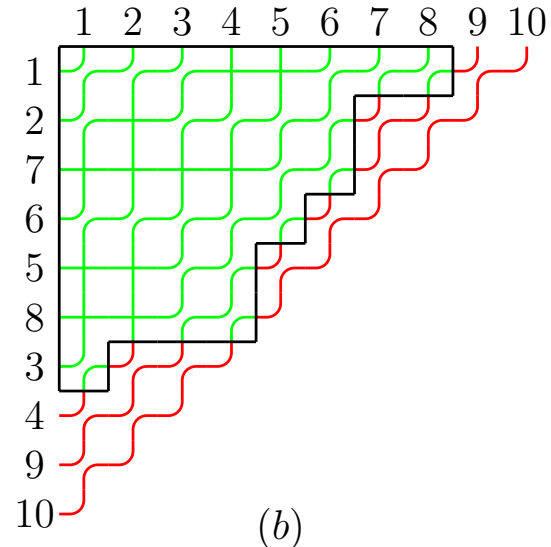

(b)

Figure 1: A 2-north-east filling of $\lambda=(8,6,6,5,4,4,1)$ and its associated reduced pipe dream.

smallest staircase containing it, and all boxes in the staircase outside of $\lambda$ are replaced by turning pipes. In other words, a $k$-north-east filling of $\lambda$ and its associated pipe dream are complementary $(0,1)$-fillings of $\lambda$ when both are identified with their sets of boxes. For example, the + 's in the pipe dream in Figure 1(b) and the marked boxes in (a) are complementary $(0,1)$-fillings of $\lambda$. The pieces in boxes outside of $\lambda$ are drawn in the pipe dream in red whereas pieces within $\lambda$ are drawn in green. We call this identification between $k$-north-east fillings of $\lambda$ and reduced pipe dreams complementary map.

For a permutation $\sigma \in \mathcal{S}_{n}$, define its (Rothe) diagram (see [Man01, Section 2.1]) to be the set of boxes in the staircase shape given by

$$
\mathrm{D}(\sigma):=\left\{\left(i, \sigma_{j}\right): i<j, \sigma_{i}>\sigma_{j}\right\} .
$$

For example, the diagram of $[1,2,7,6,5,8,3,4,9,10]$ in Figure 2 is given by the shaded area. Clearly, the number of boxes in $\mathrm{D}(\sigma)$ equals the length of $\sigma$, i.e., the minimal number of simple transpositions needed to write $\sigma$. A permutation is called dominant if its diagram is a Ferrers shape containing the box $(1,1)$. By construction, different permutations in $\mathcal{S}_{n}$ have different shapes and one can obtain every Ferrers shape in this way for some $n$. Thus, starting with a Ferrers shape $\lambda$, let $\sigma(\lambda)$ be the unique dominant permutation $\sigma \in \mathcal{S}_{n}$ for which $\mathrm{D}(\sigma)=\lambda$, where $n$ is given by the size of the smallest staircase shape containing $\lambda$. Moreover, define $\sigma_{k}(\lambda)$ to be

$$
\mathbf{1}_{k} \times \tau:=\left[1,2, \ldots, k, \tau_{1}+k, \ldots, \tau_{n}+k\right] \in \mathcal{S}_{n+k}
$$

where $\tau=\sigma(\mu)$ and $\mu$ is obtained from $\lambda$ by removing its first $k$ rows and columns. Graphically, this means that the Ferrers shape corresponding to $\sigma_{k}(\lambda)$ is obtained by removing the first $k$ columns and rows from the Ferrers shape corresponding to $\sigma(\lambda)$. Note that the north-west corner of $\sigma_{k}(\lambda)$ remains in box $(k+1, k+1)$. See Figure 2 for $\sigma_{2}(\lambda)$ with $\lambda$ as in Figure 1. The following theorem is a more precise reformulation of Theorem 1.3. 


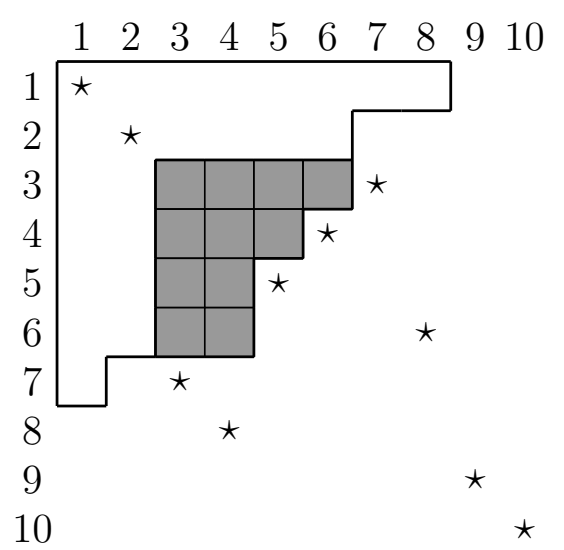

Figure 2: $\sigma_{2}(\lambda)=[1,2,7,6,5,8,3,4,9,10]$ for $\lambda=(8,6,6,5,4,4,1)$.

Theorem 2.1. Let $\lambda$ be a Ferrers shape and let $\sigma=\sigma_{k}(\lambda)$. The complementary map from $k$-north-east fillings to pipe dreams is a bijection between $\mathcal{F}_{N E}(\lambda, k)$ and $\mathcal{R} \mathcal{P}(\sigma)$.

For the proof of this theorem we use an alternative description of pipe dreams as given by A. Knutson and E. Miller in [KM05, Theorem B], or, in a more combinatorial language, by N. Jia and E. Miller in [JM08, Theorem 3]. First, we observe that the definition of antidiagonals in [JM08, Definition 2] is equivalent to the definition of a north-east chain inside $[n] \times[n]$. Following the notion in the latter, define $\mathcal{A}_{\sigma}$ for $\sigma \in \mathcal{S}_{n}$ to be the collection over all $1 \leq p, q \leq n$ of all minimal north-east chains of length $r_{\sigma}(p, q)+1$ lying inside the rectangle $[p] \times[q]$ where

$$
r_{\sigma}(p, q):=\#\{(i, j): i \leq p, j \leq q, \sigma(i)=j\} .
$$

As it can be seen in Figure 2, $r_{\sigma}(p, q)$ equals the number of stars in the matrix presentation of $\sigma$ lying inside the rectangle $[p] \times[q]$.

The set $\mathcal{R} \mathcal{P}(\sigma)$ of reduced pipe dreams for $\sigma$ can be described in terms of $\mathcal{A}_{\sigma}$ as follows. A subset of the staircase $(n-1, \ldots, 2,1)$ is a reduced pipe dream for $\sigma$ if and only if it intersects every north-east chain in $\mathcal{A}_{\sigma}$, and it is minimal in this sense. Looking at this observation in a slightly different way, we obtain the following proposition describing pipe dreams in terms of maximal fillings of the staircase shape.

Proposition 2.2. Let $\sigma \in \mathcal{S}_{n}$. A subset of the staircase $(n-1, \ldots, 2,1)$ is a reduced pipe dream for $\sigma$ if and only if its complement is a maximal filling not containing any north-east chain in $\mathcal{A}_{\sigma}$.

Proof. From the description of reduced pipe dreams above, it follows that a subset $R$ of the staircase shape is a reduced pipe dream for $\sigma_{k}(\lambda)$ if and only if it intersects every north-east chain in $\mathcal{A}_{\sigma}$, and it is minimal with respect to this property. Thus, $R$ is a reduced pipe dream if and only if its complement $R^{c}$ in the staircase shape does not contain any north-east chain in $\mathcal{A}_{\sigma}$, and $R^{c}$ is maximal with respect to this property. The latter is precisely a maximal filling of the staircase shape not containing any north-east chain in $\mathcal{A}_{\sigma}$. 
However, we do not need to consider all rectangles $[p] \times[q]$ to define $\mathcal{A}_{\sigma}$. It is enough to consider the collection of all south-east corner boxes $(p, q)$ of $\mathrm{D}(\sigma)$, each labelled by $r_{\sigma}(p, q)$. This labelled collection is called the essential set of $\sigma$ in [Man01, Section 2.2]. See Figure 4 for an example.

Proposition 2.3. $\mathcal{A}_{\sigma}$ is given by the collection of north-east chains of length $r_{\sigma}(p, q)+1$ lying inside rectangles $[p] \times[q]$ for boxes $(p, q)$ in the essential set of $\sigma$.

Proof. Every $[i] \times[i]$ rectangle for $i \leq k$, where $k$ is the smallest label of an element in the essential set of $\sigma$, contains $i$ stars. Therefore, $\mathcal{A}_{\sigma}$ does not contain any north-east chains of length smaller or equal to $k$. Moreover, observe that $r_{\sigma}(p, q)$ is constant inside a component of $\mathrm{D}(\sigma)$ and thus, among those it is enough to consider boxes $(p, q)$ in the essential set. As $r_{\sigma}(p+a, q+b)=r_{\sigma}(p, q)+a+b$ for such a box $(p, q)$, the proposition follows from the minimality condition in the definition of $\mathcal{A}_{\sigma}$.

Using this proposition, we also obtain the following description of $\mathcal{A}_{\sigma}$ coming from Ferrers shapes.

Proposition 2.4. Let $\lambda$ be a Ferrers shape and let $\sigma:=\sigma_{k}(\lambda) . \mathcal{A}_{\sigma}$ is given by the collection of all north-east chains of length $k+1$ in $\lambda$.

Proof. We have already seen that the essential set of $\sigma$ is given by the south-east corner boxes of maximal rectangles in $\lambda$ (with respect to containment) of width and height strictly larger than $k$. As all those maximal rectangles are of the form $[p] \times[q]$, the result follows with the observation that $r_{\sigma}(q, p)=k$ for such $(p, q)$.

This proposition implies the following well-known corollary.

Corollary 2.5. Every reduced pipe dream for $\sigma=\sigma_{k}(\lambda)$ lives inside $\lambda$, namely

$$
\mathcal{R P}(\sigma)=\mathcal{R} \mathcal{P}(\sigma, \lambda)
$$

Putting the arguments together, we can now prove Theorem 2.1.

Proof of Theorem 2.1. The result follows from Propositions 2.2 and 2.4.

\subsection{Generalizations to moon polyominoes}

The results in the previous section can be partially generalized to moon polyominoes which were studied by J. Jonsson in [Jon05]. A polyomino $M$ (i.e., a set of boxes in the positive integer quadrant) is called convex if for any two boxes in $M$ lying in the same row or column, all boxes in between are also contained in $M$. Moreover, $M$ is called intersection-free if for any two columns (or equivalently, rows) of $M$, one is contained in the other. A polyomino is called a moon polyomino if it is convex and intersection-free.

Without loss of generality we consider always moon polyominoes which are northwest justified, namely, they contain boxes both in the first row and in the first column. Observe that Ferrers shapes are special types of moon polyominoes. A $k$-filling of a moon 

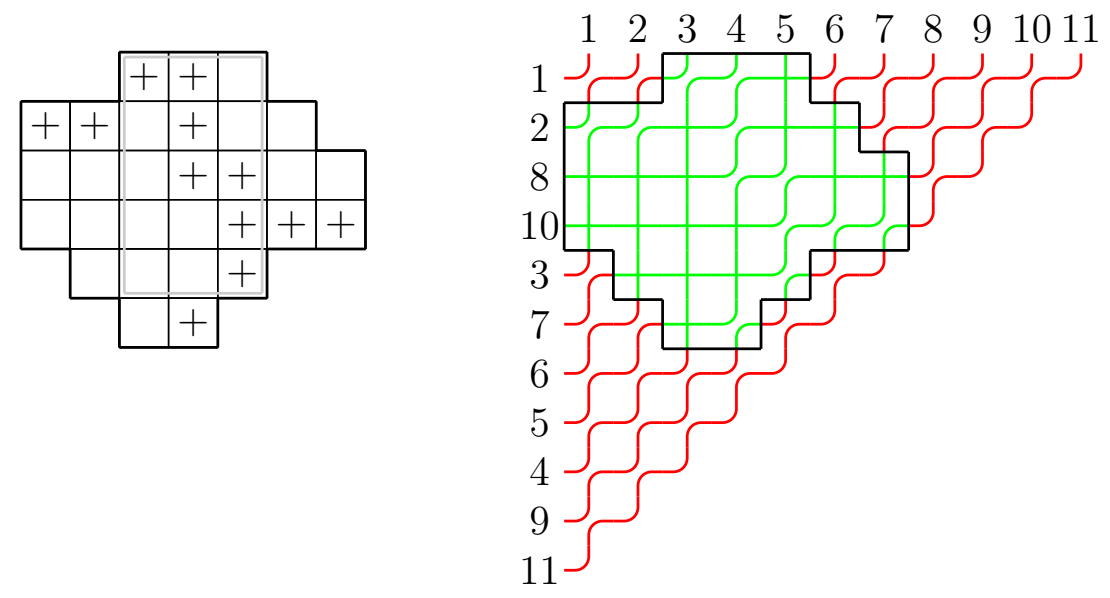

Figure 3: A 1-north-east filling of a moon polyomino and its associated pipe dream.

polyomino is defined exactly in the same way as for a Ferrers shape. See Figure 3 for an example.

To connect $k$-north-east fillings of a moon polyomino $M$ and pipe dreams of a certain permutation $\sigma=\sigma_{k}(M)$, we must relate maximal fillings of $M$ which do not contain a $(k+1)$-north-east chain in one of its maximal rectangles and maximal fillings of the staircase $(n-1, \ldots, 2,1)$ which do not contain a north-east chain of length $r_{\sigma}(p, q)+1$ in any rectangle $[p] \times[q]$. Define $\sigma_{k}(M)$ as follows: for a maximal rectangle $R$ in $M$ of width and height both strictly larger than $k$, let $(a+1, b+1)$ and $(i, j)$ be its north-west and south-east corner boxes. Label the box $(i+b, j+a)$ with $a+b+k . \sigma_{k}(M)$ is the permutation with this collection as its essential set. This means that the diagram $\mathrm{D}(\sigma)$ of $\sigma$ has $(i+b, j+a)$ as a south-east corner with labels $r_{\sigma}(i+b, j+a)=a+b+k$. Using [Man01, 2.2.8], it is easy to see that this construction is well defined. Note that maximal rectangles of width or height less than or equal to $k$ cannot contain any north-east chain of length larger than $k$ and thus do not contribute to the essential set of the corresponding permutation. For example, the moon polyomino $M$ in Figure 3(a) has maximal rectangles $(a+1, b+1)-(i, j)$ given by

$$
\begin{array}{cc}
(1,3)-(5,5), & (1,3)-(6,4), \\
(3,1)-(4,7), & (2,2)-(5,5), \\
(2,1)-(4,6),
\end{array}
$$

where the first maximal rectangle is highlighted. Thus, for $k=1$, the resulting essential set and the associated diagram can be seen in Figure 4, and the associated permutation is $\sigma_{1}(M)=[1,2,8,10,3,7,6,5,4,9]$. As all maximal rectangles in a Ferrers shape are of the form $[p] \times[q]$, the definition of $\sigma_{k}(\lambda)$ reduces in this case to the definition given in the previous section. Moreover, observe that in the more general context of moon polyominoes which are not Ferrers shapes, Corollary 2.5 does not hold.

The following theorem is a more precise reformulation of Theorem 1.4. 


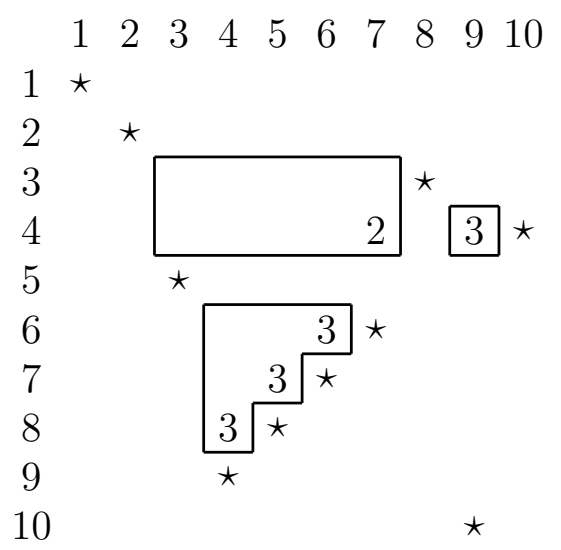

Figure 4: The essential set and the diagram $\mathrm{D}(\sigma)$ for $\sigma=[1,2,8,10,3,7,6,5,4,9]$.

Theorem 2.6. The complementary map from $k$-north-east fillings of a moon polyomino $M$ to pipe dreams of $\sigma=\sigma_{k}(M)$ is a bijection between $\mathcal{F}_{N E}(M, k)$ and $\mathcal{R} \mathcal{P}(\sigma, M)$.

Proof. Recall that the set $\mathcal{A}_{\sigma}$ is the collection over all $(p, q)$ in the essential set of $\sigma$ of all minimal north-east chains in $[p] \times[q]$ of length $r_{\sigma}(p, q)+1$. By construction, every such $(p, q)$ comes from a maximal rectangle $R$ in $M$ with north-west corner $(a+1, b+1)$ and south-east corner $(i, j)$. Thus, $(p, q)=(i+b, j+a)$ and $r_{\sigma}(p, q)=a+b+k$.

As $M$ is intersection-free by definition, no box strictly south-west or strictly north-east of $R$ is contained in $M$. Therefore, any $(k+1)$-north-east chain inside $R$ can be extended to a $(a+b+k+1)$-north-east chain inside $[p] \times[q]$. This implies that a $(k+1)$-north-east chain inside $R$ cannot be contained in the complement of a pipe dream for $\sigma$ living inside $M$. In total, we obtain that the set of complements of pipe dreams for $\sigma$ living inside $M$ are exactly maximal fillings of $M$ not containing a north-east chain of length $k+1$. This completes the proof.

We now use this theorem together with the main theorem in [Jon05] to get new insights on pipe dreams. A stack polyomino is a moon polyomino where every column starts in the first row. Let $S$ be a stack polyomino and let $\lambda$ be the Ferrers shape obtained from $S$ by properly rearranging the columns. J. Jonsson proved in [Jon05, Theorem 14] that the number of $k$-north-east fillings of $S$ with a given number of + 's in every row equals the number of $k$-north-east fillings in $\lambda$ with the same number of + 's in every row. Moreover, he conjectured that this property still holds if the stack polyomino $S$ is replaced by a moon polyomino. Therefore, we obtain the following corollary and the conjecture for the analogous statement for moon polyominoes.

Corollary 2.7. Let $S$ be a stack polyomino and let $\lambda$ be the associated Ferrers shape. The number of pipe dreams in $\mathcal{R} \mathcal{P}\left(\sigma_{k}(S), S\right)$ with a given number of crossings in every row is equal to the number of pipe dreams in $\mathcal{R} \mathcal{P}\left(\sigma_{k}(\lambda)\right)$ with the same number of crossings in every row. 


\subsection{The simplicial complex of north-east-fillings}

We are now in position to prove Corollary 1.5. The canonical connection between $k$-northeast fillings and reduced pipe dreams can be used in the same way as described in the proof of [Stu10, Corollary 1.3] for $k$-triangulations in this more general setting. For the necessary background on simplicial complexes and in particular on subword complexes, we refer to [KM04]. A box in a moon polyomino $M$ is called passive if it is not contained in any north-east chain in $M$ of length $k+1$. Let $\Delta(M, k)$ be the simplicial complex with vertices being the collection of boxes in $M$, and with facets being $k$-north-east fillings of $M$.

Corollary 2.8. $\Delta(M, k)$ is the join of a vertex-decomposable, triangulated sphere and a full simplex of dimension $i-1$, where $i$ equals the number of passive boxes in $M$. In particular, it is shellable and Cohen-Macauley.

Proof. Label the box $(i, j)$ by $i+j-1$. The simplicial complex $\Delta(M, k)$ is precisely the subword complex for the permutation $\sigma_{k}(M)$ and the word given by the labels of all boxes in $M$ (where $i$ and the simple transposition $s_{i}$ are identified) read row by row from east to west and from north to south. Observe that the passive boxes are exactly those boxes which are contained in all facets of $\Delta(M, k)$. Thus, the corollary follows from Theorem 2.1 together with Theorems 2.5 and 3.7 in [KM04].

\subsection{A mutation-like operation on pipe dreams}

Generalizing the notion in the previous section, one can define a pure simplicial complex $\Delta(\sigma)$ for any $\sigma \in \mathcal{S}_{n}$ by defining the facets as the complements in the staircase of reduced pipe dreams in $\mathcal{R} \mathcal{P}(\sigma)$ (see [KM04]). Using the property that two pipes in a reduced pipe dream $D$ cross at most once, one can define a mutation-like operation on facets of $\Delta(\sigma)$ as follows. One can mutate the facet $F(D)$ of $\Delta(\sigma)$ associated to $D$ at a vertex $b$ if the two pipes in $D$ which touch in $b$ cross somewhere else. In other words, one can mutate $F(D)$ at a vertex $b$ if the starting points $i<j$ of the two pipes in $D$ which touch in $b$ form an inversion of $\sigma$. The mutation of $F(D)$ at such a vertex $b$ is then defined to be the facet $F\left(D^{\prime}\right)$ for the reduced pipe dream $D^{\prime}$ such that

(i) the two turning pipes in $b$ are replaced in $D^{\prime}$ by two crossing pipes,

(ii) the unique crossing $b^{\prime}$ of those two pipes is replaced in $D^{\prime}$ by two turning pipes.

By construction, the pipe dream $D^{\prime}=(D \cup b) \backslash b^{\prime}$ is again in $\mathcal{R} \mathcal{P}(\sigma)$ and thus its complement $F\left(D^{\prime}\right)=(F(D) \backslash b) \cup b^{\prime}$ forms another facet of $\Delta(\sigma)$.

\section{From pipe dreams to south-east fillings}

In this section we describe a bijection between pipe dreams for $\sigma_{k}(\lambda)$ and $k$-south-east fillings of $\lambda$, for a Ferrers shape $\lambda$. For the sake of readability, we do this construction in 


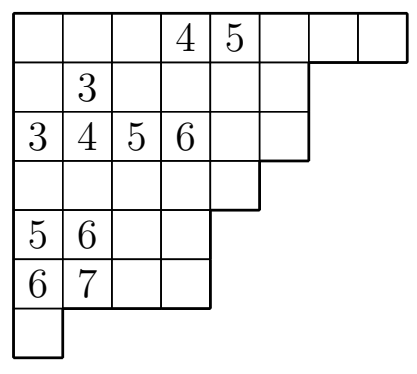

$$
\begin{aligned}
& \left(\begin{array}{lllllllllll}
1 & 1 & 2 & 3 & 3 & 3 & 3 & 5 & 5 & 6 & 6 \\
5 & 4 & 3 & 6 & 5 & 4 & 3 & 6 & 5 & 7 & 6
\end{array}\right)
\end{aligned}
$$

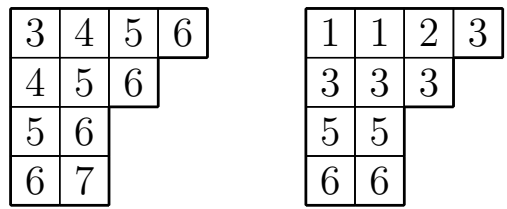

Figure 5: Labelling of the crossing boxes in the pipe dream in Figure 1(b), the corresponding compatible sequence, and its insertion and recording tableau.

several steps. A similar approach was described by S. Fomin and A. Kirillov [FK97], and in the particular case of the permutation $[1, n, n-1, \ldots, 3,2]$ by A. Woo [Woo04].

\subsection{From pipe dreams to flagged tableaux}

Define a $k$-flagged tableau as a semistandard tableau in which the entries in the $i$-th row are smaller than or equal to $i+k$, and denote the set of $k$-flagged tableaux of shape $\lambda$ by $\mathcal{F} \mathcal{T}(\lambda, k)$. These were introduced by M. Wachs [Wac85], where she proves that the Schubert polynomial of a vexilliary permutation is equal to a flagged Schur function (see also work of V. Reiner and M. Shimozono [RS95, Theorem 24]). We now present a bijection between the set $\mathcal{R} \mathcal{P}(\sigma)$ of reduced pipe dreams of $\sigma=\sigma_{k}(\lambda)$ and the set $\mathcal{F} \mathcal{T}(\mu, k)$ of $k$-flagged tableaux of shape $\mu=\mathrm{D}(\sigma)$. Note that the permutations that can be obtained as $\sigma_{k}(\lambda)$ for some Ferrers shape $\lambda$ are precisely those that fix the first $k$ integers, and apply a dominant permutation on the rest. These belong to a bigger family of permutations called vexilliary permutations. The bijection we present in this section can be found in more generality in the work of C. Lenart [Len04, Section 4] for vexilliary permutations, but we include the full description in this particular case for the sake of completeness. For more on flagged tableaux and their connections to geometry, see, e.g., [KMY09].

For a reduced pipe dream $D \in \mathcal{R} \mathcal{P}(\sigma)$ with $\sigma$ being of length $\ell$, define the reading biword to be the $2 \times \ell$ array by reading $\left(\begin{array}{c}i \\ i+j-1\end{array}\right)$ for every crossing box $(i, j)$ in $D$ row by row from east to west and from north to south. See Figure 5 for an example. It is known (and easy to check) that this gives a bijection between $\mathcal{R} \mathcal{P}(\sigma)$ and the set of compatible sequences $\mathcal{C S}(\sigma)$, defined by S. Billey, W. Jockush and R. Stanley in [BJS93] as the set of all $2 \times \ell$ arrays of the form $\left(\begin{array}{c}a_{1}, \ldots, a_{\ell} \\ b_{1}, \ldots, b_{\ell}\end{array}\right)$ satisfying the following properties:

1. $a_{1} \leq a_{2} \leq \cdots \leq a_{\ell}$,

2. if $a_{i}=a_{i+1}$, then $b_{i}>b_{i+1}$,

3. $b_{1} b_{2} \cdots b_{\ell}$ is a reduced word for $\sigma$, where $i$ denotes the simple transposition $s_{i}=$ $(i, i+1)$, and

4. $a_{i} \leq b_{i}$. 
One can see from the definition that a compatible sequence $t$ for $\sigma$ can be written as the concatenation $t=t_{1} \cdots t_{m}$, where $t_{i}=\left(\begin{array}{c}i^{\left|w_{i}\right|} \\ w_{i}\end{array}\right)$, and $w_{i}$ is decreasing. Observe that $\sigma$ fixes all $j \leq k$ and thus, every letter in $w_{i}$ is larger than or equal to $\max (i, k)$.

Define a map $\mathcal{C S}(\sigma) \rightarrow \mathcal{F} \mathcal{T}(\mu, k)$ as follows. Let $t \in \mathcal{C} \mathcal{S}(\sigma)$ be a compatible sequence for $\sigma$. Insert the letters of the word formed by the bottom row of $t$ using column EdelmanGreene insertion [EG87] into a tableau, while recording the corresponding letters from the first row. This produces an insertion tableau $P(t)$ and a recording tableau $Q(t)$. The image in $\mathcal{F} \mathcal{T}(\mu, k)$ is now defined to be $Q(t)$. To prove that this is a well defined bijection, we need two preliminary lemmas (see [Len04, Section 4]).

Lemma 3.1. For a ferrers shape $\lambda$, all insertion tableaux $P(w)$ for reduced words $w$ of $\sigma=\sigma_{k}(\lambda)$ are equal. The shapes of $P(t)$ and $Q(t)$ are given by $\mu=D(\sigma)$.

Proof. Since $\mu$ is a Ferrers shape where the north-west corner is located in box $(k+$ $1, k+1$ ), $P(t)$ only depends on $\sigma$ and not on the actual compatible sequence (see [Man01, Section 2.8.3]). Moreover, the labelling $i+j-k-1$ for $(i, j) \in \mu$ gives a reduced expression for $\sigma$ which column inserts into itself (see [Man01, Remark 2.1.9]). Therefore, the shapes of $P(t)$ and $Q(t)$ are both given by $\mu$.

Lemma 3.2. Let $\sigma=\sigma_{k}(\lambda)$ for a Ferrers shape $\lambda$ and let $t=t_{1} t_{2} \cdots t_{m}$ be a compatible sequence for $\sigma$. Every letter in $w_{i} \cdots w_{m}$ is strictly larger than $j$ if and only if every letter in the first $j-k$ rows of $Q(t)$ is strictly less than $i$.

Proof. Every letter in $w_{i} \cdots w_{m}$ is strictly larger than $j$ if and only if all of the occurrences of $1,2, \ldots, j$ in $w=w_{1} \cdots w_{m}$ appear in $w_{1} \cdots w_{i-1}$. Since $w$ is a reduced word for $\sigma$, this is equivalent to saying that the first $j$ letters of the permutation given by $w_{1} \cdots w_{i-1}$ are the same as those in $\sigma$, when written in one line notation. Since $\mu=\mathrm{D}(\sigma)$ is a Ferrers shape where the north-west corner is located at the box $(k+1, k+1)$, this is equivalent to saying that every entry on the first $j-k$ rows of $Q(t)$ and of $Q\left(t_{1} \cdots t_{i-1}\right)$ coincide. As $Q\left(t_{1} \cdots t_{i-1}\right)$ contains only letters strictly smaller than $i$, the result follows.

Putting the connections between reduced pipe dreams, compatible sequences and flagged tableaux together, we obtain the following theorem.

Theorem 3.3. Let $\sigma=\sigma_{k}(\lambda)$ for a Ferrers shape $\lambda$, and let $\mu=D(\sigma)$. The map sending $D$ in $\mathcal{R P}(\sigma)$ to the recording tableau of the reading biword of $D$ is a bijection between $\mathcal{R} \mathcal{P}(\sigma)$ and $\mathcal{F} \mathcal{T}(\mu, k)$.

Proof. It is left to show that the map sending a compatible sequence $t$ to $Q(t)$ is a well defined bijection between $\mathcal{C S}(\sigma)$ and $\mathcal{F} \mathcal{T}(\mu, k)$. Let $t \in \mathcal{C S}(\sigma)$. By Lemma 3.1, $Q(t)$ has shape $\mu$, and by Lemma 3.2 with $i=\ell+k+1$ and $j=\ell+k$, every letter in row $\ell$ in $Q(t)$ is less than or equal to $\ell+k$. Thus, $Q(t)$ is indeed a $k$-flagged tableau. Furthermore, the construction is bijective, since Edelman-Greene insertion can be inverted to obtain $t$.

An example of the bijection can be seen in Figure 5. 

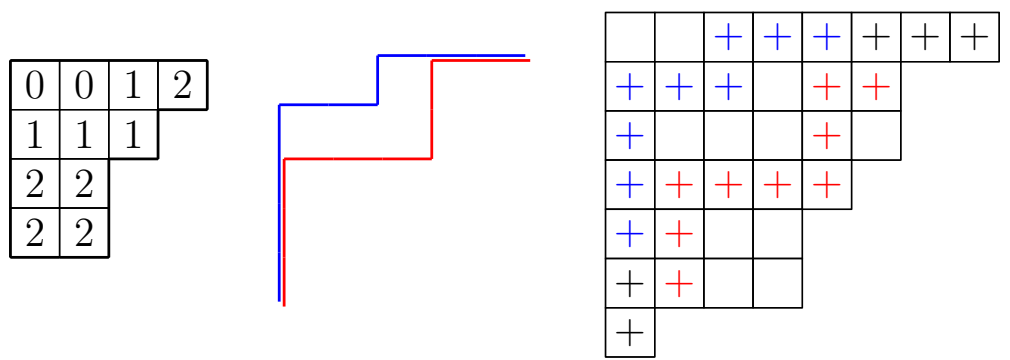

Figure 6: Reverse plane partition corresponding to Figure 5 and its corresponding 2-fan of paths.

\subsection{A cyclic action on flagged tableaux}

In this subsection we define a cyclic action on $k$-flagged tableaux. The flagged promotion $\rho(Q)$ of a $k$-flagged tableau $Q$ is defined as follows.

(i) Delete all the instances of the letter 1 ,

(ii) apply jeu de taquin to the remaining entries,

(iii) subtract 1 from all the entries,

(iv) label each empty box on row $i$ with $i+k$.

One can easily see that $\rho(Q)$ is indeed a $k$-flagged tableau, since the empty boxes after step (iii) must form a horizontal strip, which means there is at most one empty box per column. Furthermore, as every box gets moved at most up by one row, and at the end one subtracts 1 from all the entries, the tableau obtained after step (iii) is $k$-flagged as well. The argument is finalized with the observation that if one adds a horizontal strip in which every box gets added its maximum possible value, the tableau is still $k$-flagged, since the row-weakness is assured by the maximality of the value of the entries on each row, and the column-strictness is assured by the fact that the entries in row $i-1$ are all strictly less than the maximal value on row $i$.

\subsection{From flagged tableaux to fans of paths and south-east fillings}

We proceed as in [FK97] to obtain a reverse plane partition of height $k$ from a $k$-flagged tableau. Let $\lambda$ be a Ferrers shape and let $\mu=\mathrm{D}\left(\sigma_{k}(\lambda)\right)$. Since every entry in row $i$ of a $k$-flagged tableau of shape $\mu$ is less than or equal to $i+k$ and greater than or equal to $i$ (as the tableau is semistandard), one can subtract $i$ from all the entries in row $i$, for all rows, and obtain a reverse plane partition of shape $\mu$ and height $k$ (i.e., where the only possible entries are $0,1, \cdots, k$. From this plane partition, one can obtain a $k$-fan of noncrossing north-east paths inside $\mu$, simply by letting the $i$-th path be the boundary between the entries labelled $i$ and $i+1$. To obtain a bijection between $k$-flagged tableaux of shape $\mu$ and the set $\mathcal{F}_{S E}(\lambda, k)$ of $k$-south-east fillings of the shape $\lambda$, one lifts the $i$-th 
path from the bottom by $i-1$ and turns it into a path of + 's inside $\lambda$. This is done by top left justifying the $k$-th path inside $\lambda$, placing the beginning of the $k-1$-th path one box below and to the right than the beginning of the $k$-th path, and continuing in this fashion. As a final step, one adds a + in all the boxes which are in the same column and below the beginning of each path, and in those in the same row and to the right of the end of each path. See Figure 6 for an example; the red marks come from the red path, the blue from the blue path, and the additional black marks are the extra marks we have added, which are contained in any 2-south-east filling.

Putting the described bijections together, we obtain Theorem 1.2.

Theorem 3.4. Let $\lambda$ be a Ferrers shape. The composition of the described maps is a bijection between $\mathcal{F}_{N E}(\lambda)$ and $\mathcal{F}_{S E}(\lambda)$.

As mentioned in the introduction, $k$-triangulations of the $n$-gon can be seen as $k$-northeast fillings of the staircase shape $(n-1, \ldots, 2,1)$, and $k$-fans of Dyck paths of length $2(n-$ $2 k$ ) can be seen as $k$-south-east fillings of the same staircase (see e.g. [Kra06a, Rub06]). Thus, we obtain Theorem 1.1. See Figure 7 for an example.

Corollary 3.5. In the case where $\lambda$ is the staircase shape $(n-1, \ldots, 2,1)$, the described map is a bijection between $k$-triangulations of the $n$-gon and $k$-fans of noncrossing Dyck paths of length $2(n-2 k)$.

\section{Properties of fillings and $k$-triangulations}

Using Theorem 2.6, we obtain several properties of $k$-north-east fillings of moon polyominoes and of Ferrers shapes and $k$-triangulations in particular. Some of them were already known while others where only conjectured.

The first property was proved in the case of stack polyominoes by J. Jonsson in [Jon05, Theorem 10]. It follows immediately from Theorem 2.1.

Corollary 4.1. Every $k$-north-east filling of a moon polyomino $M$ contains $i$ boxes, where $i$ equals the total number of boxes in $M$ minus the length of $\sigma_{k}(M)$. In particular, $i$ equals the number of boxes in the first $k$ rows and columns in the case of Ferrers shapes.

The second property is part of the main theorem in [PS09, Theorem 1.4(i)] and concerns the star property as described as well in [Stu10]; for the notion used here, we refer as well to the latter.

Corollary 4.2. Every $k$-triangulation of the $n$-gon consists of exactly $n-2 k$-stars.

Proof. This follows from the description of $k$-triangulations in terms of $k$-north-east fillings of the staircase shape. As in this case $\sigma$ is given by $\mathbf{1}_{k} \times[n-2 k, \ldots, 1]$, we obtain $2 k$ outer pipes, as well as $n-2 k$ inner pipes connecting $i$ with $i$ for $k<i \leq n-k$, and which contains exactly $2 k+1$ turns. See Figure 7 for an example. This is exactly the star property in [Stu10] and thus completes the proof. 

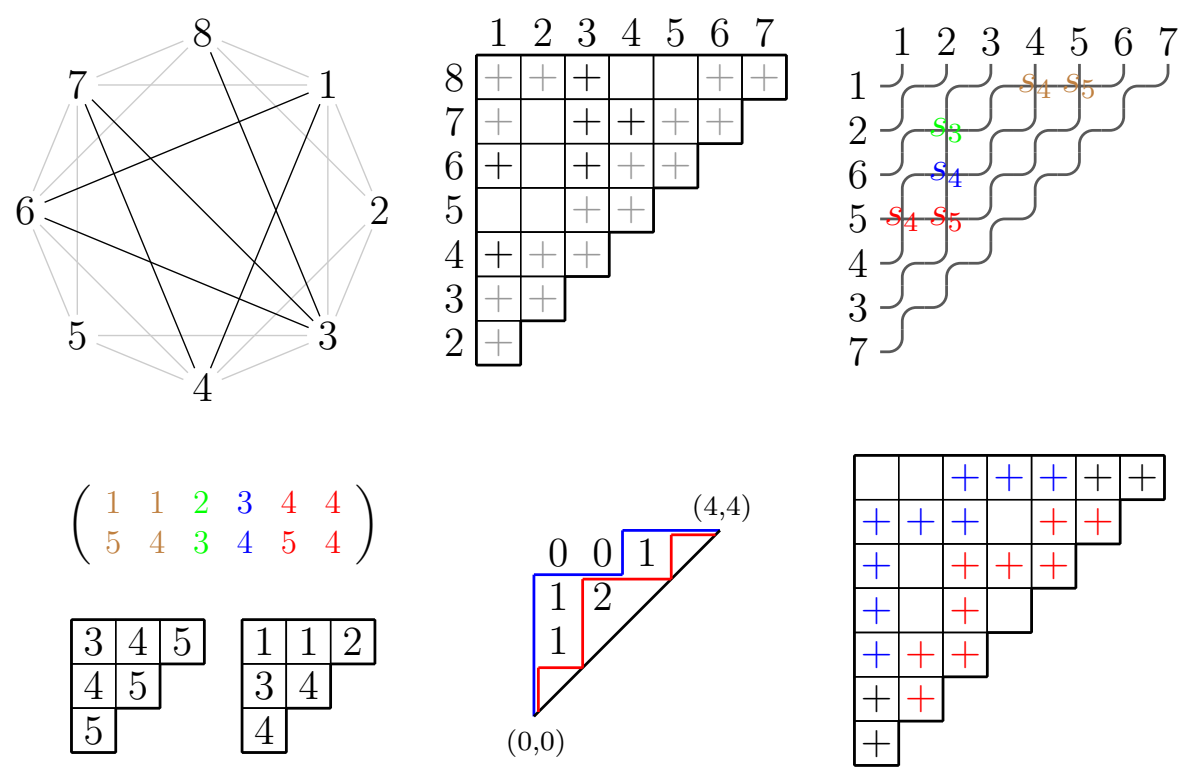

Figure 7: An example of all the steps in the bijection for the case of the staircase $\lambda=$ $(7, \ldots, 1)$ and $k=2$ : a 2-triangulation of the 8 -gon, the 2-north-east filling of $\lambda$, the pipe dream for $[1,2,6,5,4,3,7,8]$, the compatible sequence, the insertion and recording (2-flagged) tableau of shape $(3,2,1)$, the reverse plane partition of shape $(3,2,1)$, the 2 -fan of Dyck paths of length 8, and finally the 2-south-east filling of the $\lambda$.

Using the description of mutations for $k$-triangulations in Section 2.4, one can also describe the mutation of a facet in the simplicial complex

$$
\Delta_{n, k}:=\Delta\left(\mathbf{1}_{k} \times[n-2 k, \ldots, 1]\right) .
$$

This mutation corresponds to removing a diagonal in a $k$-triangulation and replacing it by the unique other diagonal which gives a $k$-triangulation. This operation is called fip in [PS09, Theorem 1.4(iii)].

Corollary 4.3. A facet $F$ in the simplicial complex $\Delta_{n, k}$ can be mutated at any vertex $d=(i, j) \in F$ for which $k<|i-j|<n-k$.

Proof. The inversions of $\sigma=\mathbf{1}_{k} \times[n-2 k, \ldots, 1]$ are given by all $(i, j)$ for which $k<i<$ $j \leq n-k$. Thus, all $n-2 k$ inner pipes in $D \in \mathcal{R} \mathcal{P}(\sigma)$ mutually cross. It follows from Section 2.4 that the facet corresponding to $D$ can be mutated at any vertex $(i, j)$ for which $k<|i-j|<n-k$.

The next property of the constructed bijection will allow us to obtain a refined counting of $k$-triangulations, as conjectured by C. Nicolas [Nic09]. Note that a diagonal $(i, j)$ for which $|i-j| \leq k$ or $|i-j| \geq n-k$ is contained in every $k$-triangulation of the $n$-gon. Thus, we define the degree of a vertex $i$ as the number of vertices $j$ adjacent to $i$ for which $k<|i-j|<n-k$. 
Theorem 4.4. The degree of vertex 1 in a k-triangulation is equal to the number of touching points of the lowermost Dyck path of its corresponding $k$-fan of Dyck paths with the main diagonal (excluding the beginning and ending points of the path). Furthermore, each edge $(1, j)$ corresponds to the touching point with coordinates $(j-k-1, j-k-1)$.

Proof. Let $t$ be the compatible sequence corresponding to a $k$-triangulation $T$, decomposed into $t_{1} \cdots t_{m}$, where $t_{i}=\left(\begin{array}{c}i\left|w_{i}\right| \\ w_{i}\end{array}\right)$ as in the definition. By construction, there is a diagonal $(1, j)$ if and only if the column $\left(\begin{array}{l}n+1-j \\ n+1-j\end{array}\right)$ does not appear in $t$. By virtue of this, and the fact that each letter in $w_{i}$ is larger than or equal to $i$, we have that every letter in $w_{n+1-j} \cdots w_{m}$ is strictly larger than $n+1-j$. By Lemma 3.2 with $i=n-j+1$, every letter in the first $n+1-j-k$ rows of $Q(T)$ is smaller than or equal to $n-j$. In particular, every letter in row $n+1-j-k$ of the corresponding reverse plane partition is strictly smaller than $k$. By construction, this implies that the lowermost path touches the diagonal at the point $(j-k-1, j-k-1)$. The converse follows clearly from the argument.

Figure 7 shows an example for $k=2$, where vertex 1 is connected to vertices 4 and 6 , and the (red) lowermost Dyck path touches the diagonal at positions

$$
\begin{aligned}
& (1,1)=(4-2-1,4-2-1) \text { and } \\
& (3,3)=(6-2-1,6-2-1) .
\end{aligned}
$$

As described in [Nic09], we use this theorem to prove Conjecture 2 therein. For an explicit expression for the determinant, we refer to [Kra06b, Theorem 4].

Corollary 4.5. The number of $k$-triangulations of a convex $n$-gon having degree $d$ in a given vertex is given by the determinantal expression

$$
\operatorname{det}\left(\begin{array}{cccc}
\operatorname{Cat}_{n-2 k} & \cdots & \text { Cat }_{n-k-2} & B_{n-k-1}^{k}(d) \\
\vdots & \ddots & \vdots & \vdots \\
\operatorname{Cat}_{n-k-1} & \cdots & \text { Cat }_{n-3} & B_{n-2}^{k}(d)
\end{array}\right),
$$

where $\mathrm{Cat}_{\ell}$ is the usual Catalan number, and where $B_{\ell}^{k}(d)=\frac{2 k+d-3}{\ell}\left(\begin{array}{c}2 \ell-2 k-d+2 \\ \ell-1\end{array}\right)$.

\subsection{Rotation of the $n$-gon and a CSP for flagged tableaux}

There is a natural cyclic action $\rho$ on $k$-triangulations given by rotating the vertex labels in the $n$-gon counterclockwise. The following conjecture is due to V. Reiner [Rei09].

Conjecture 4.6 (V. Reiner). Let $\lambda$ be the staircase shape $(n-1, \ldots, 2,1)$ and let $k$ be a positive integer. The triple

$$
\left(\mathcal{F}_{N E}(\lambda, k),\langle\rho\rangle, F(q)\right)
$$

exhibits the cyclic sieving phenomenon (CSP) as described in [RSW04], where

$$
F(q):=\prod_{1 \leq i \leq j<n-2 k} \frac{[i+j+2 k]_{q}}{[i+j]_{q}}
$$

is a natural q-analogue of the cardinality of $\mathcal{F}_{N E}(\lambda, k)$. 
We can describe the cyclic action on $k$-triangulations induced by rotation in terms of flagged promotion, as defined in Section 3.2.

Theorem 4.7. The constructed bijection maps the cyclic action on $k$-triangulations to the cyclic action given by flagged promotion on flagged tableaux.

Proof. Let $T$ be a triangulation with compatible sequence $t$, and recording tableau $Q=$ $Q(t)$. First, we describe the compatible sequence $\rho(t)$ of $\rho(T)$, then we describe the compatible sequence for the flagged promotion $\rho(Q)$, and finally we show that they are the same.

Note that any non-edge $(i, j)$ in $T$, with $i<j$, gets encoded in the compatible sequence as a column $\left(\begin{array}{l}n+1-j \\ n+i-j\end{array}\right)$. In particular, the non-edges $(i, n)$ get encoded as $\left(\begin{array}{l}1 \\ i\end{array}\right)$. For $j<n$, the switch from the non-edge $(i, j)$ in $T$ to $(i+1, j+1)$ in $\rho(T)$ corresponds to turning the column $\left(\begin{array}{l}n+1-j \\ n+i-j\end{array}\right)$ in $t$ into the column $\left(\begin{array}{c}n-j \\ n+i-j\end{array}\right)$ in $\rho(t)$. Likewise, the switch from the non-edge $(i, n)$ in $T$ to $(1, i+1)$ in $\rho(T)$ corresponds to turning the column $\left(\begin{array}{l}1 \\ i\end{array}\right)$ into the column $\left(\begin{array}{c}n-i \\ n-i\end{array}\right)$, and placing it in the right place to make sure $\rho(t)$ is a compatible sequence.

Let $\tilde{Q}$ be the ordinary promotion of $Q$. It is well-known by the relationship between promotion and Edelman-Greene insertion that $\tilde{Q}$ is the recording tableau of the biword obtained by turning each column of the form $\left(\begin{array}{l}i \\ j\end{array}\right)$ into the column $\left(\begin{array}{c}i-1 \\ j\end{array}\right)$, for $2 \leq i \leq n$, and turning each column of the form $\left(\begin{array}{l}1 \\ j\end{array}\right)$ into one of the form $\left(\begin{array}{c}n \\ n-j\end{array}\right)$, and placing them at the end of the compatible sequence in reverse order. This is the same transformation as described in the above paragraph, except for columns $\left(\begin{array}{l}1 \\ j\end{array}\right)$ which got mapped into columns $\left(\begin{array}{c}n-j \\ n-j\end{array}\right)$ instead. We now proceed to slide these columns of the form $\left(\begin{array}{c}n \\ n-j\end{array}\right)$ towards the position of the column $\left(\begin{array}{l}n-j \\ n-j\end{array}\right)$ one by one, starting from the left while adjusting the element in the top row accordingly, and show that this transforms $\tilde{Q}$ into $\rho(Q)$. Notice that every letter in the bottom row between these two columns is greater than or equal to $n-j+2$, which means they all commute with $n-j$. Thus, the bottom row is still a reduced word for $\sigma$. Lemma 3.1 implies that that all reduced expressions for $\sigma$ have the same insertion tableau. Since we now record $n-j$ instead of $n$ for those columns, this procedure transforms $\tilde{Q}$ into $\rho(Q)$.

Using this connection, we obtain the following corollary.

Corollary 4.8. Conjecture 1.7 is equivalent to Conjecture 4.6.

\section{A positivity result for Schubert polynomials}

In this section we use the results about moon polyominoes in Section 2.2 to obtain a property of Schubert polynomials. It was shown in [FK96] that Schubert polynomials are a generating series for pipe dreams, more precisely, for a permutation $\sigma$,

$$
\mathfrak{S}_{\sigma}\left(x_{1}, \ldots, x_{n}\right)=\sum_{D \in \mathcal{R} \mathcal{P}(\sigma)} \prod_{(i, j) \in D} x_{i} .
$$

We obtain the following theorem and thus Corollary 1.6. 
Theorem 5.1. Let $S$ be a stack polyomino, let $\lambda$ be the associated Ferrers shape and let $k$ be a positive integer. Then

$$
\mathfrak{S}_{\sigma_{k}(S)}\left(x_{1}, x_{2}, \ldots\right)-\mathfrak{S}_{\sigma_{k}(\lambda)}\left(x_{1}, x_{2}, \ldots\right)
$$

is monomial positive. In particular, $\mathfrak{S}_{\sigma_{k}(S)}(1,1, \ldots)$ is greater than or equal to the number of $k$-flagged tableaux of shape $\lambda$.

Proof. This follows from Corollary 2.7.

\section{Acknowledgements}

The authors would like to thank Christian Krattenthaler for pointing to a conjecture in [Nic09] proved in Corollary 4.5, and an anonymous referee for helpful comments on the manuscript. The first author would also like to thank Sergey Fomin and David Speyer for helpful discussions.

\section{References}

[BB93] N. Bergeron and S. Billey, RC-graphs and Schubert polynomials, Experiment. Math. 2 (1993), no. 4, 257-269.

[BJS93] S. Billey, W. Jockush, and R. Stanley, Some combinatorial properties of Schubert polynomials, J. Algebraic Combin. 2 (1993), 344-374.

[EG87] P. Edelman and C. Greene, Balanced tableaux, Adv. Math. 63 (1987), no. 1, 42-99.

[Eli07] S. Elizalde, A bijection between 2-triangulations and pairs of non-crossing Dyck paths, J. Combin. Theory Ser. A 114 (2007), no. 8, 1481-1503.

[FK96] S. Fomin and A. Kirillov, The Yang-Baxter equation, symmetric functions, and Schubert polynomials, Discrete Math. 153 (1996), 123-143.

[FK97]_, Reduced words and plane partitions, J. Algebraic Combin. 6 (1997), no. $4,311-319$.

[JM08] N. Jia and E. Miller, Duality of antidiagonals and pipe dreams, Sém. Lothar. Combin. 58 (2008), B58e.

[Jon05] J. Jonsson, Generalized triangulations and diagonal-free subsets of stack polyominos, J. Comb. Theory, Ser. A 112 (2005), 117-142.

[KM04] A. Knutson and E. Miller, Subword complexes in Coxeter groups, Adv. Math. 184 (2004), no. 1, 161-176.

[KM05] _ Gröbner geometry of Schubert polynomials, Ann. of Math. 161 (2005), no. 3, 1245-1318.

[KMY09] A. Knutson, E. Miller, and A. Yong, Gróbner geometry of vertex decompositions and of flagged tableaux, J. Reine Angew. Math. 630 (2009), 1 - 31. 
[Kra06a] C. Krattenthaler, Growth diagrams, and increasing and decreasing chains in fillings of Ferrers shapes, Adv. in Appl. Math. 37 (2006), 404-431.

[Kra06b] _ Watermelon configurations with wall interaction: exact and asymptotic results, J. Physics Conf. Series 42 (2006), 179-212.

[Len04] C. Lenart, A unified approach to combinatorial formulas for Schubert polynomials, J. Algebraic Combin. 20 (2004), 263-299.

[Man01] L. Manivel, Symmetric functions, Schubert polynomials and degeneracy loci, SMF/AMS Texts and Monographs 6 (2001).

[Nic09] C. Nicolás, Another bijection between 2-triangulations and pairs of non-crossing Dyck paths, in DMTCS as part of the FPSAC 2009 conference proceedings (2009).

[Pil10] V. Pilaud, Multitriangulations, pseudotriangulations, and some problems of realization of polytopes, $\mathrm{PhD}$ thesis, Université Paris 7 and Universidad de Cantabria (2010).

[PP10] V. Pilaud and M. Pocchiola, Multitriangulations, pseudotriangulations and primitive sorting networks, preprint, available at arXiv:abs/1009.5344 (2010).

[PS09] V. Pilaud and F. Santos, Multitriangulations as complexes of star polygons, Discrete Comput. Geom. 41 (2009), no. 2, 284-317.

[Rei09] V. Reiner, personal communication.

[RS95] V. Reiner and M. Shimozono, Key polynomials and a flagged LittlewoodRichardson rule, J. Combin. Theory Ser. A 70 (1995), no. 1, 107-143.

[RSW04] V. Reiner, D. Stanton, and D. White, The cyclic sieving phenomenon, J. Combin. Theory Ser. A 108 (2004), 17-50.

[Rub06] M. Rubey, Increasing and decreasing sequences in fillings of moon polyominoes, to appear in Adv. in Appl. Math., available at arXiv:math/0604140 (2006).

[Sta11] R.P. Stanley, Catalan Addendum, http://www-math.mit.edu/ rstan/ec/ catadd.pdf (2011), version 30 April 2011.

[Stu10] C. Stump, A new perspective on k-triangulations, J. Combin. Theory Ser. A 118 (2010), no. 6, 1794-1800.

[Wac85] M. Wachs, Flagged Schur functions, Schubert polynomials, and symmetrizing operators, J. Combin. Theory Ser. A 40 (1985), no. 2, 276-289.

[Woo04] A. Woo, Catalan numbers and Schubert polynomials for $w=1(n+1) \ldots 2$, preprint, available at arXiv:math/0407160 (2004). 